\title{
Development Plan for the Nucleon Physics Laboratory Facility at LAMPF
}

\author{
J. B. McClelland \\ A. Bacher* \\ R. L. Boudrie \\ T. A. Carey \\ J. Donahue \\ C. D. Goodman** \\ M. W. McNaughton \\ N. Tanaka \\ O. B. van Dyck \\ R. Werbeck
}

\section{DISCLAIMER}

This report was prepared as an account of work spnnsored by an agency of the United States Government. Neither the United States Government nor any agency thereof, nor any of their employees, makes any warranty, express or implied, or assurnes any legal liability or responsibility for the accuracy, completeness, or usefulness of any information, apparatus, prociuct, or process disclosed, or represents that its use would not infringe privately owned rights. Reference herein to any specific commercial product, process, or service by trade name, trademark, manufacturer, or otherwise does not necessarily constitute or imply its endorsement, recommendation, or favoring by the Uriited States Government or any agency thereof. The views and opinions of authors expressed herein do not necessarily state or reflect those of the United States Government or any agency thereof. 


\section{DEVELOPMENT PLAN FOR THE NUCLEON PHYSICS LABORATORY FACILITY AT LAMPF}

by

J. B. McClelland, A. Bacher, R. L. Boudrie, T. A. Carey, J. Donahue, C. D. Goodman, M. W. McNaughton, N. Tanaka, O. B. van Dyck, and R. Werbeck

\section{ABSTRACT}

A 3- to 4-year plan is described for upgrading the LAMPF Nucleon Physics Laboratory including a neutron timeof-flight facility for the $(p, n)$ reaction, a medium-resolution spectrometer for $\left(p, p^{\prime}\right)$ and $(n, p)$ studies, and a dedicated facility for atomic beam, cudies. Development of these facilities and relationships to other ongoing developments are detailed. The scope of the new physics programs supporied by such a facility is discussed.

\section{INTRODUCTION}

In January 1984, the Program Advisory Subcommittees for the High Resolution Proton Spectrometer (HRS) and Nucleon Physics Laboratory (NPL) at LAMFF requested "a review of facility developments that would enhance LAMPF's capabilities in the $\mathrm{H}^{-}$beam areas." In response, we have assembled in this report several ideas covering NPL additions that would benefit both the nucleon and nuclear physics programs at LAMPF. We discuss a neutron time-of-flight facility (NTOF) for $(p, n)$ studies, a medium-resolution spectrometer (MRS) for ( $p, p^{\prime}$ ) and $(n, p)$ stuties, an atomic beam facility, and the relationships among these possible NPL changes to other additions such as a new polarized $\mathrm{H}^{-}$("P ") source, a 100-ns beam buncher, and a new polarized targer. The full, several-milliondollar upgrade is viewed as a 3- to 4-year plan, although some components could be available much sooner. We briefly describe each facet of the ungrade and 
present an overview of some of the new physics available in the nuclear charge-exchange reactions. A report similar to this one was presented to the August 1984 joint HRS/NPL Program Advisory Subcommittees with high priority recommendation for the new ion source and the MRS. It also recommended a buncher in 1986 on the existing source to benefit both the nucleon-nucleon (N-N) and $(p, n)$ nuclear physics programs. The Technical Advisory Panel was also presented with the proposal for the MRS and NTOF facilities in October 1984. Their recommendation was that both of these facilities be implemented soon, NTOF in 1986 and MRS in 1987. Subsequent presentations have been made to the LAMPF Board of Directors, DOE, and LAMPF Users Group. In December 1984, a two-day workshop was held to discuss the two facilities. Approximately 50 attendees representing 20 universities and national laboratories participated in that worl:shop. This proposal represents the consensus of opinion of that workshop as to the requirements needed to pursue the NTOF and MRS programs.

\section{FACILITY UPGRADE}

A. $(p, n)$ Facility

One of the earliest possible additions to the NPL could be a beam swinger and neutron time-of-flight (NTOF) facility for ( $p, n)$ reactions.

The primary $\mathrm{H}^{-}$proton beam is deflected from its current direction in Area $B$ by approximately 30 degrees by a switching magnet (Fig. 1) into the swinger area. Quadrupole magnets maintain focusing requirements on the $(p, n)$ target as the swinger angle is varied. Beamline instrumentation such as wire chambers, ionization chambers, and polarimeter for determining bean profile, intensity, and polarization will be used similar to existing devices on other $\mathrm{P}^{-}$beamlines.

The beam swinger is a five magnet system used to vary the incident angle of the proton beam on the $(p, n)$ target, effectively changing the neutron scattering angle while keeping the neutron flight path and detectors stationary. This system is described in more detail in Appendix A. As currently envisioned, this system will allow for momentum transfers greater than $3 \mathrm{fm}^{-1}$ at all energies between 200 and $800 \mathrm{MeV}$. The fourth magnet of the swinger restores the proton beam into a beam dump capable of stopping and shielding up to $600 \mathrm{nA}$ of $800-\mathrm{MeV}$ protons. The existing Area $B$ beam dump meets these requirements. The swinger, $(p, n)$ target, and beam dump reside in a shielded crypt appended to the Area $B$ room as shown in Fig. 1. Not shown in that figure is the neutron spin precession system used to precess longitudinal polarized neutrons into transverse so as to be measurable in the neutron polarimeter, and also to periodically reverse the outgoing neutron spin for cancellation of numerous systematic effects 
associated with polarimetry measurements away from 0 degrees. This precession system is being developed by $P$ and MP Divisinns for use at Indiana University Cyclotron Facility (IUCF) in 1986 and consists of a dipole magnet followed by a superconducting solenoid. This configuration meets all spin direction requirements. Suitable collimation before and after the neutron spin precession system produces a neutron beam matched to the detector size and distance from the $(p, n)$ target .

A flight path of up to 600 meters is possible with some excavation as shown in Fig. 2. Given the expected timing performance of both the accelerator and detectors, this will yield neutron energy resolutions of 1 to $2 \mathrm{MeV}$ at $800 \mathrm{MeV}$. This is consistent with $(p, n)$ work being carried out at other laboratories at lowe: energies. Beam timing characteristics will be monitored using inductively coupled beam pickoffs as used at Weapons Neutron Research (WNR). These have been tested in Line $C$ during the 1985 production period. Fencing along the flight path would restrict stray personnel from the neutron beam. Experience at IUCF and WNR as well as dose calculations indicate that the neutron flux is low enough to allow for experimentalists to be in the detector hut with the beam on. Additionally, some shielding may be possible. Suitable instrumentation will assure that the primary proton beam cannot reach the detector station. Additional access to the staging area and Area A counting houses will be provided. Infrequent interruptions for large equipment access through the fenced flight path ale acceptable. Note that the flight path passes over the current access road to Area $C$ by 15-20 feet, allowing for continual access during experiments.

The detector hut is probably a $3 \times 10$ meter ${ }^{2}$ portable house on a carriage so that it can be positioned at three or four predetermined locations along the 600 -meter flight path. It would be a self-contained unit housing the detectors and their support systems, electronics, and computer acquisition area. Alternatively, the acquisition station could be separate but move in tandem with the detector station. It would require utility and patching stations at each of the three or four acquisition stations.

The polarimeter for these studies is being developed by groups in $P$ and MP Divisions for use at IUCF. The polarimeter, sketched in Fig. 3, has three planes (with the possible addition of an additional analyzer plane), each $1 \mathrm{~m}$ long by $1 \mathrm{~m}$ high by $10 \mathrm{~cm}$ thick. Each plane consists of 10 individual cells $1 \mathrm{~m}$ long by $10 \mathrm{~cm}$ high by $10 \mathrm{~cm}$ thick. The cells contain liquid scintillator, providing an ideal active analyzer for polarimetry based on ${ }^{3} \mathrm{H}(\mathrm{n}, \mathrm{n})^{1} \mathrm{H}$ elastic scattering. Time differences between the two ends of a cell give a position 
REPFODUCEO FOCM
BEST
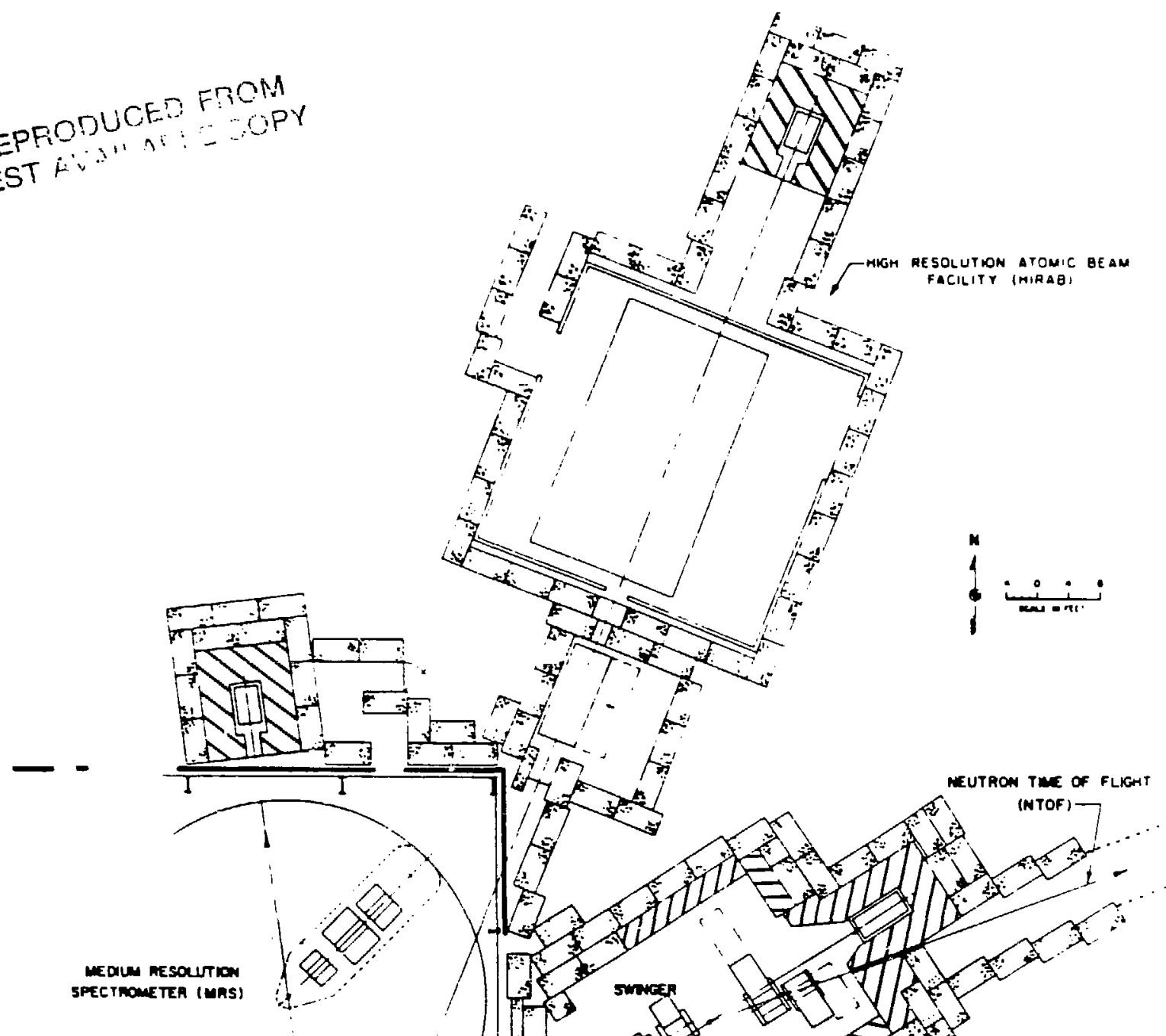

\section{AREA "B"}

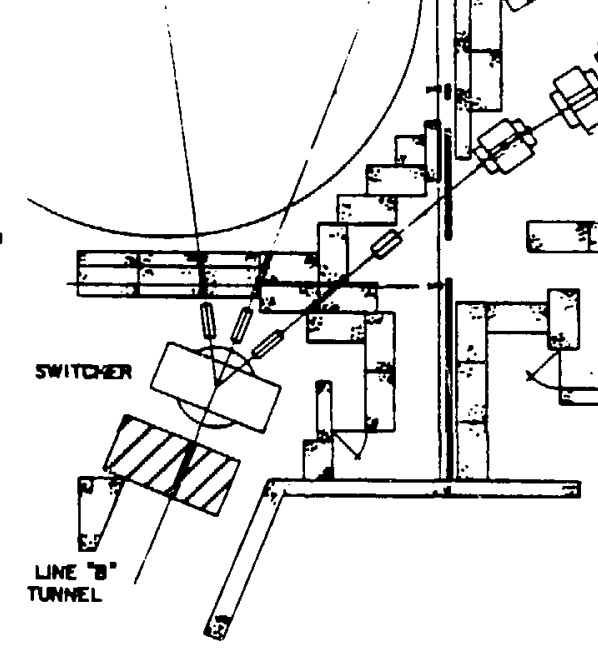

Proposed modifications of NPL area. 
REPRODU!ner ran: BE:-

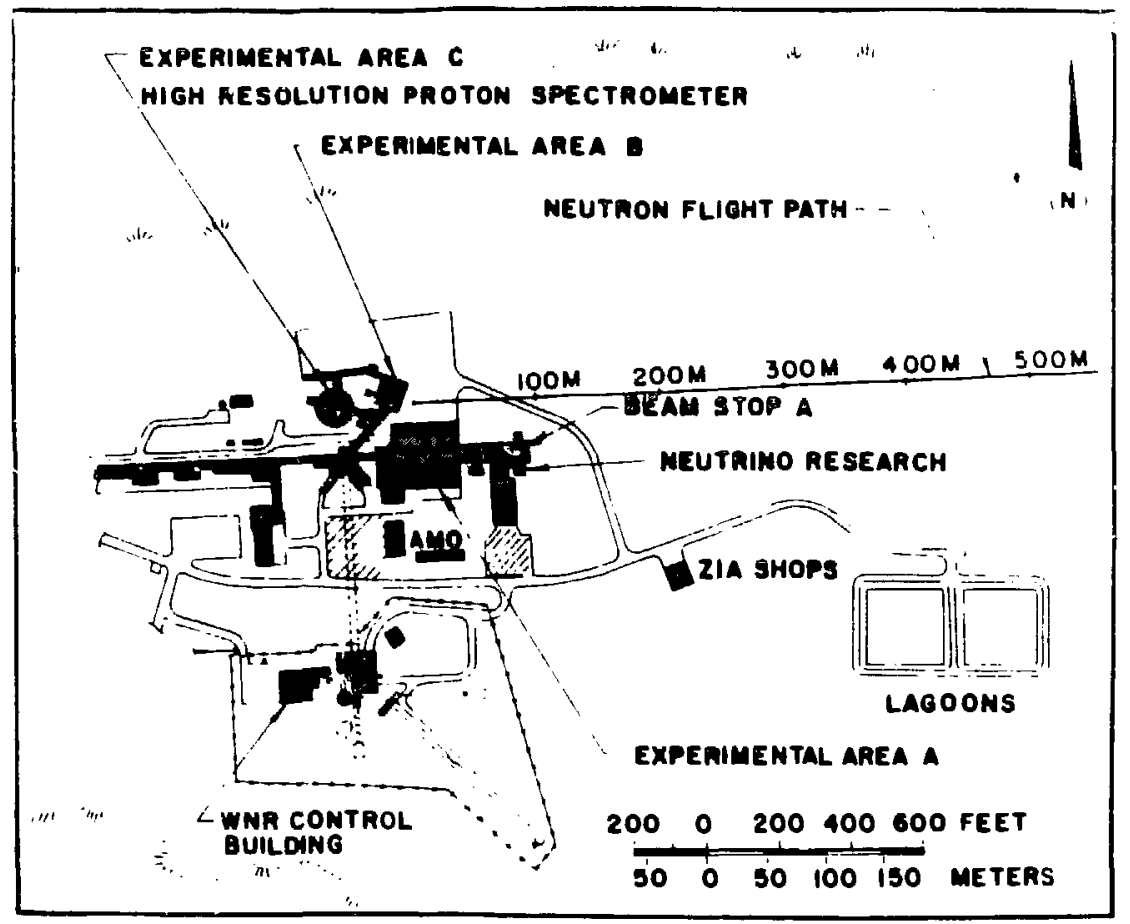

Fig. 2

Layout of NPL area showing neutron time-of-flight (NTOF) path and end station.

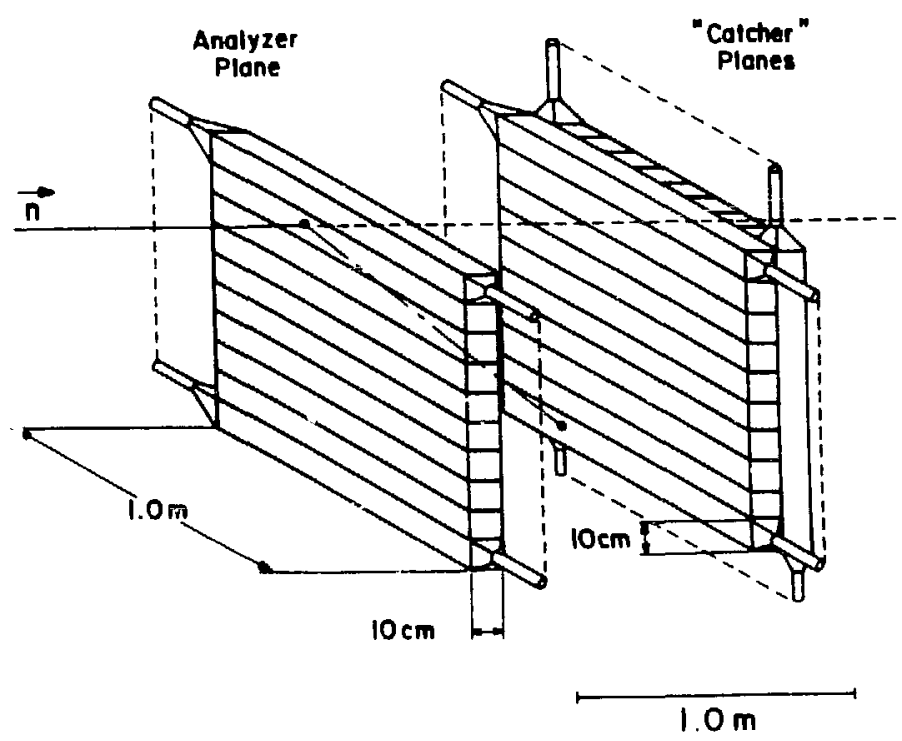

Fig. 3

Schematic of $(\vec{p}, \vec{n})$ neutron polarimeter. 
resolution of $\$_{5} \mathrm{~cm}$ along the cell length. Vertical coordinates are obtained from the cell number.

From these two sets of coordinates, polar and azimuthal second-scattering angles are calculated, and both transverse polarization compnnents at the polarimeter are simultaneously analyzed in a manner similar to that used in the HRS system. By suitable spin precession of the neutrons hetween the target and polarimeter, all three final-state polarization components originating from the target may be measured. A more detailed discussion of the polarimeter can be found in the appendices of LAMPF Research Proposal 881. Due to their modular design, these detectors are also well suited to cross-section measurements, which will undoubtedly be the first set of measurements made at the new facility. These detectors will likely be used at IUCF during 1986 and 1987. At the end of that period they can be brought back to LAMPF for use as par: of the NTOF facility. Due to the low duty cycle at LAMPF, different electronics will be needed from that used at IUCF, specifically buffered electronics similar to the HRS system to acquire more than one event within a macropulse. A minicomputer running standard LAMPF software will acquire data at the remote station.

The spin-precessor capabilities now present in Area B would allow for spin-transfer measurements to be supported compatibly with HRS. We expect that a large emphasis would be placed on spin-transfer measurements in the $(\vec{p}, \vec{n})$ reaction at the same energies currently considered "HRS Prime," which means a more efficient use cf low-energy time.

\section{B. Medium-Resolution Spectrometer (MRS)}

A medium-resolution spectrometer, $\Delta E \sim 1 \mathrm{MeV}$, would have several roles. It would complement the HRS with high acceptance where $1-\mathrm{MeV}$ resolution is adequate, possibly serving about hale the HRS queue; serve as a home base for polarized-target and light-nuclei experiments, which cannot exploit the HRS energy resolution; and serve as a detector for $(n, p)$ studies with resolution well matched to the narrowest available neutron spectrum. The MRS would occupy the present Area $B$ area most of the time.

Design work has proceeded for the optical per ormance of such a spectrometer. The design goals that were considered are listed in Table I. Since the instrument will be used to measure the polarization of the scattered particles, the net angle of deflection should be small, ideally about $15^{\circ}$. This will insure that for all momenta considered, there will be no "dead spots" in the polarization analyzed (i.e., the outgoing polarization is not precessed significantly into the longitudinal component at the focal plane detectors). One would also like the focal plane angle to be small so that to first order 
only the position information in the first wire chamber plane is needed to make missing mass cuts on the data before complete data acquisition and analysis. The demand for large solid angle and reasonable resolution taken together with the small net deflection angle and focal plane angle is extremely hard to meet.

Four different layouts were tried, each with a large number of variations:

a) QQDQQ system

b) QDQ system with strung negative n-value

c) QD(-D) system with strong wedge focussing

d) QDS system with strong negative n-value.

QQDQQ System

The original work on this system was done without regard to the small focal plane angle requirement. A solution which, at first glance, looked acceptable was presented to the workshop meeting in Los Alamos on December 17, 1984. The major aberration term for $x$-focussing is $(x / \theta)$ except for the central momentum. The intention was to use measurements of position and angle to correct for the first order as well as higher order aberrations. There turned out to be some difticulties with this procedure. The problem is that for $\Theta=25 \mathrm{mrad}$, and larger, the dispersion is very low. Apparently the combination of deflections in the quadrupoles and in the rather weak dipole produces a nondispersive system for part of the solid angle, and of course for a limited momentum range.

QDQ System

In this system the median-plane focussing is made strong enough by use of a negative $n$-value in the dipole. The tapering of the pole faces naturally needs to be quite strong and the nominal field therefore has to be kept low to avoid saturation in the poles.

QDS System

In an attempt to make a system with a normal focal plane angle, a system with a sextupule was tried. It was found that the sextupole strength in the dipole and sextupole was such that it was impossible to accept a vertical angle of $40 \mathrm{mrad}$. For acceptance angles of $\pm 20 \mathrm{mrad}$ the results were reasonable.

$\mathrm{QD}(-\mathrm{D})$ system

The total angle of deflection should not exceed $20^{\circ}$ and this does not produce much net focussing, hence the need for quadrupoles. An alternative is to use two dipoles with opposite field directions. This will increase the dipole contribution to the focussing and perhaps also make it easier to produce a smaller focal-plane angle. In a particular design considered, the dipole bend angles were $33^{\circ}$ and $15^{\circ}$. The entrance and exit pole face angles were rather 
large to produce strong enough focussing, but the sextupole terms are not impractically large.

The QD(-D) system is the most promising and a layout is shown in Fig. 4. Further work has proceeded to fill the acceptance with random rays and directly investigate the performance in the plane of the wire chambers. Acceptance studies have been investigated for large target spot sizes $\left(-25 \mathrm{~cm}^{2}\right)$ for the case of the $(n, p)$ capability. Additional requirements to be cddressed are improved small-angle scattering coverage, localized target shielding, generalized second arm, focal plane polarimeter, and a nonmagnetic pivot to accommodate a polarized target. The spectrometer facility will require a permanent counting house.

\section{TABLE I}

MRS DESIGN REQUIREMENTS

Maximum Momentum:

Angular Range:

Overall Length:

FULL ACCEPTANCE

Momentum Acceptance:

Resolution:

Solid Angle:

REDUCED ACCEPTANCE

Momentum Acceptance:

Resolution:

Solid Angle:

Angular Resolution:
$1900 \mathrm{MeV} / \mathrm{C}$

$800 \mathrm{MeV}$ deuteron

9 m

$\begin{array}{ll} & @ 800 \mathrm{MeV} \\ \pm 12 \% & \pm 150 \mathrm{MeV} \\ 0.4 \% & 5 \mathrm{MeV} \\ 15 \mathrm{msr} & \end{array}$

$\pm 1.5 \%$

$\pm 20 \mathrm{MeV}$

$0.04 \%$

$0.5 \mathrm{MeV}$

$10 \mathrm{msr}$

$\pm 2 \mathrm{mrad}$

Once the spectrometer construction is complete, it would be used to service $\left(p, p^{\prime}\right)$ requirements. During that time the effects of large target area associated with the $(n, p)$ reaction would be studied. The initial implementation of a 


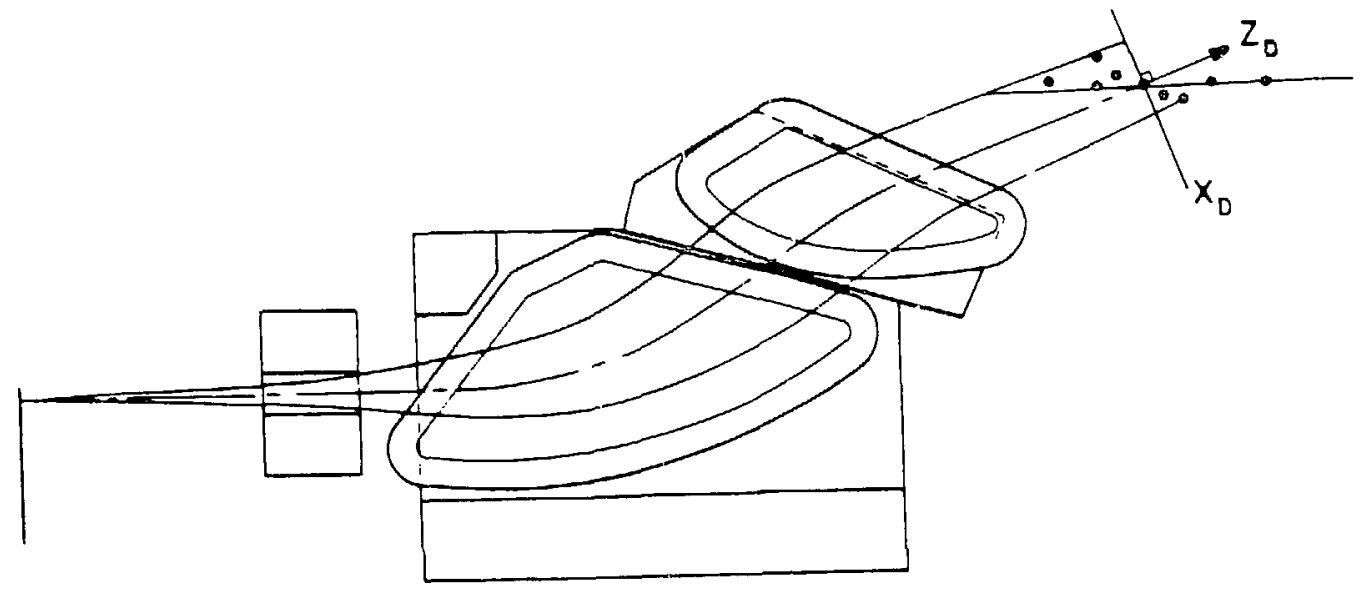

Fig. 4

Layout for QD(-D) system of MRS.

neutron source for $(n, p)$ experiments might best be done using the existing neutron channel in Room $B R$, (BR) replacing the $L D_{2}$ target with a thin lithium target. This would produce a nuclear physics grade neutron beam with an energy width less than $1 \mathrm{MeV}$. This implies moving the MRS between Area $B$ and BR, which is an advantage from another point of view. It would allow the MRS to be used in nucleon-nucleon programs in BR for which a keen interest was demonstrated during the December workshop. Since the wall separating Area $B$ and BR is movable, it would be reasonable to move the MRS on air pads between the two areas on a cycle basis. Details of the $(n, p)$ application of the MRS can be found in LAMPF Research Proposal 823. Once the initial phase of the (n,p) program in BR is completed, a decision can be made as to the best targeting scheme for the program in general. A possibility exists to target the proton beam in Area $B$ about 2 meters upstream of the (n,p) target then dogleg it around the MRS and restore $\dot{t}$ in the Area $B$ dump. This is similar to the system being developed at Tri-Universities Meson Facility (TRIUMF) for a similar application at lower energies. More information about shielding problems should be available at that time.

Future studies involving polarization transfer might use the rather large values of $D_{L L}$ for $\mathrm{Li}(\sim 0.5$ at $800 \mathrm{MeV})$ in conjunction with neutron spin precession and the MRS focal-plane polarimeter. However, the reduced counting rates associated with polarimeter efficiencies may require further development. This 
facility--a polarized, monoenergetic neutron beam, a proton spectrometer, and a polarimeter-would be a powerful and unique tonl for spin-isospin nurleat physics, highly complementary to the $(\vec{p}, \vec{n})$ program being proposed as well as to the $\left(\vec{p}, \vec{p}^{\prime}\right)$ program at HRS.

C. High Resolution Atomic Beam Facility

The prime goal of the Neutral Particle Beam (NPB) Program is to assess the feasibility of using neutral particle beams as directed energy weapons outside the earth's atmosphere. This initiative calls for a significantly enhanced program of technological feasibility demonstrations. The High Resolution Atomic Beam Facility (HIRAB) will serve as an extremely valuable resource as the research and technology demonstration of beam sensing and control. A wide range of basic atomic physics issues can also be investigated to an unprecedented precision.

In addition to demonstrations that accelerator technology can meet the beam specifications of a NPB weapon, experiments are needed to demonstrate directional sensing and control of the NPB as a part of a beam delivery system. Several methods have been proposed frr this purpose with Laser Resonance Florescence (LRF) being the preferred technique because it can sense the entire neutral beam nondestructively with wide bandwidth at a precision limited by the divergence of the NPB itself. This method can also be used in multiple arrangements to make measurements on various portions of the beam to provide beam focus information.

The project will provide for the construction of a 1600 square foot airconditioned building. A 20 -foot by 40 -foot by 4 -foot thick concrete pad, isolated from the building slab, will provide vibration isolation for the laser and optics. A movable beam plug upstream of the facility will allow it to be occupied while the other areas are being used. The lack of "hands-on" access has been a problem with previous experiments.

There are numerous advantages to doing such experiments at LAMPF. A new $\mathrm{H}^{-}$ ion source for use with the Proton Storage Ring (PSR) results in higher peak currents. The beam energy at LAMPF is variable from 200 to $800 \mathrm{MeV}$ in small steps. An active experimental program of basic atomic physics research using $\mathrm{H}^{0}$ and $\mathrm{H}^{-}$beams currently exists. Most of the beamline components to prepare a high-quality $\mathrm{H}^{-}$beam exist. Groups with expertise in pariicle beam preparation, laser optics, photoexcitation, and particle and laser beam characterization are on site at Los Alamos. LAMPF has been used previously for NPB experiments and procedures are established. 
LAMPF has received DOE approval to schedule application-oriented NPB experiments that do not interfere with overall IAMPF operations. Such experiments can be allocated beam following suitable advance requests and approval by the LAMPF management. However, NPB requests for resources, including dedicated accelerator operations, requiring support that is not otherwise needed for the nuclear physics program, must be reimbursed. Atomic physics basic research proposals will continue to follow the traditional route through the Program Advisory Committee (PAC).

D. Beamline Modifications

In order to use the MRS, NTOF, and iiRAB iacilities, a delivery system must be built which allows beam to be transported to each of these facilities. It has been decided to magnetically deflect the beam to the MRS and NTOF areas using a high-field dipole bending magnet; HIRAB will use the undeflected beam. The high magnetic field prevents the transport of negatively charged hydrogen ions (polarized and unpolarized) to either of these facilities because of magnetic stripping. HIRAB will be able to use either $\mathrm{P}^{-}$or $\mathrm{H}^{-}$beam. Also, only one of the three facilities will be able to run at any given time. With one excepticn, whenever one of the three areas is accepting beam, the other two areas will be able to be occupied by personnel; the exception is that personnel will be excluded from the MRS when HIRAB is using the beam.

The External Proton Beamline (Area B) delivers the proton beam to the magnetic "switcher" referred to above. That portion of the beamline which is presently located in the Line $B$ tunnel will not be Eundamentally changed, although stripper mechanisms and a polarimeter will be added. The Lire $B$ tunnel will be extended into Area B (the building which presently houses "Room BR" and "Area B"); i.e., a shielded "caboose" will be added to house the switching magnet, some beam diagnostic apparatus, and movable beam plugs which will be used for personnel safety considerations.

The dipole magnet which served as the magnetic element of the University of Colorado 52" Cyclotron has been procured and will be used as the magnetic switcher in the NPL upgrade. It will deflect stripped $\mathrm{P}^{-}$(or $\mathrm{H}^{-}$) beams of up to $800 \mathrm{MeV}$ in kinetic energy $29^{\circ}$ to the left into the MRS facility which is to be located in the existing Area $B$ building. It will also be used to deflect the beam $28^{\circ}$ to the right into another bending magnet (an existing, surplus dipole) which will bend the beam an additional $7^{\circ}$ to the right into the new NTOF swinger crypt to be built east of Area $B$. The undeflected beam will be able to pass through the MRS shield enclosure into the new HIRAB building which is to be constructed northeast of Area B (at the present Area B beamstop location). 
Three sets of beam plugs will de fabricated and placed at the exit ports of the "switcher"; the ports will extend to MRS, NTOF, and HTRAR.

The switching magnet and beam plugs will be enclosed in a minimum ihickness concrete house; the minimum amount of shielding is to be used to reduce the amount of floor space lost to the MRS in the building. This eifort to maximize floor space for MRS will prevent the installation of phase space tailoring collimators for MRS and NTOF because of the additional snace required for collimators and additional shieldirg installation. Future collimator installation may be carried out at the cost of reduced angular range for MPS.

Once beyond the Line $B$ tunnel extension, each of the three transport lines will contain veam diagnostic apparatus, a cauadrupole doublet (in each case, an existing, surplus doublet), and a vacuum systom. Each beamiine will ultimately terminate in a beam stop capable of stopping several hundred nanoamfs of protons.

The MRS shield enclosure will be lightly shielded along its sides and vill have no shield roof above it. Thus, even thin MRS targets will need to be iocally shielded in order to run even a few nanoamps of beri into them. The HIRAB shield enclosure will also be thinly shielded along its walls and wiIl have no shield roof. However, HIRAB will intercept the proton beam with only a laser beam; thus, not much shielding will be required. The swinger crypt for the NTOF facility will be a relatively well-shielded enclosure (including a shielded roof) which will permit several hundred nanoamps of protons to strike targets up to one gram thick. The crypi will be quite large $(12 \mathrm{~m} \times 7.5 \mathrm{~m})$ to accommodate the motion of the large swinger magnets; much of the shielding will be reused surplus shielding from the University of Colorado 52" Cyclotron facility and from Area $A$ of IAMPF.

There will be separate Run Permissive (RP) and Personnel Safety Systems (PSS) for the MRS, NTOF, and HIRAB facilities. The NTOF PSS will be split into the swinger crypt PSS and several sections of flight path PSS. The swinger crypt is the shielded enclosure described above, but the neutron time-of-flight path will be a long (several hundred meters long), narrow (from three to five meters wide) fenced-in region which will contain the ccllimated neutron beam. Details of the flight path PSS need to be finalized, including the detector area and the region of the existing access road east of the Line $A$ beam stop area which will have the neutron beam passing above it.

In addition to running beam into the three areas noted above, provisions have been made to allow low intensity ( 10 picoamps) experiments to be carried out upstrean of the HIRAB facility in the existing Area $B$ building. Therefore, 
if necessary, experiments could be run in a manner similar to the way in which they are presently carried out in Area B. During such periods, the MRS would have to be rotated out of the way of the experimental setups, and access to the MRS would be prohibited. However, at those times, access to the HIRAB facility would be permitted upon insertion of a massive movable beam siop into the path of the beam; this movable beam stop is to be constructet just upstream of the HIRAB facility.

During the 1986 shutdown (January to mid-June 1986) it is planned to install the switching magn.t and the three movable beam plug assemblles located immediately dowristream of it. Then, the shielded "caboose" acidition to the Line B ilnnel will be constructed around the magnet and beam plugs. If possible, the modifications to the Area $B$ building needed for the completed NPL upgrade project will be started (e.g., addition of personnel access doors and rr.ioval/relocation of electrical breaker panels and transformers). The transport line to be used in delivering beam to the HIRAB facility (which will be built during the shutciown) will be assembled. Work will continue in preparation for the installation of the transport ?ines to be used in delivering veins to the MRS and NTOF swinger. It is planned to carry out a physics program in the present Area B room, ard begin the HIRAB experimertal program during the 1986 production run. Ir has not been decided if the swilching magriet will be used during this time. This is all part of a staged approach toward impleilenting the NPL upgrade. 
Switching magnet preparations:

(support structure, magnet

modifications, vacuum)

Line B "caboose" shielding:

concrete

40

penetrations

5

Beam plug assemblies:

25

TOTAL

100

E. Time Table and Costs

Table II shows some interconnections among these NPL modifications and other facility additions such as the new polarized ion source ( 1 uA average at full duty), a 100-ns beam burcher for neutron timing, and a new polarized target. 
Date

Modification

Now $\mathrm{P}^{-}$Source

$\mathrm{P}^{-}$Buncher

Swinger

Lithium

Target in BR

MF.S

Addi :ional

Polarized

Target
Programs

\begin{tabular}{|c|c|c|c|c|}
\hline $\overrightarrow{\mathrm{n}}+\mathrm{N}$ & $\overrightarrow{\mathrm{p}}+\mathrm{H}^{\vec{t}}$ & $(\vec{p}, \vec{p})$ & $(n, p)$ & $(\vec{p}, \vec{n})$ \\
\hline a & & (i) & (c) & a \\
\hline$a$ & & & (b) & $\mathrm{a}$ \\
\hline
\end{tabular}

a

(b) $\begin{array}{lll}\text { a } & \text { a } & \text { a }\end{array}$

a a

a Means the equipment is of primary benefit to the program.
$b_{\text {Indicates a secondary benefit. }}$
$c_{\text {Of primary benefit when }(\vec{n}, \vec{p}) \text { program begins. }}$

A beam buncher on the existing Lamb-shift polarized ion source would permit additional neutron-nucleon experiments to proceed $\left[\right.$ e.g., $\left.\Delta \sigma_{L}(n, p)\right]$ as early as 1986. If NTOF comes on-line earlier than the new $P^{-}$sucurce, several options exist in the commissioning of the facility, including bunched unpolarized beam. These options allow some flexibility in the exact time when the new source must be operational since it allows important programs to proceed in the interim.

The time scale for the NTOF system for $(p, n)$ cross-section measurements is estimated to be mid 1987. This assumes a 100-ns buncher in 1986. The system would transport the beam to the $(p, n)$ targeting system, the full 600-meter flight path, detector hut with P/MP divisions' detectors, electronics, and computer. The cost of this minimai system is given in Table IIIa. Costs are based on using existing magnets, P/MP divisions' detectors, and a replacement beam dump. Some parts of beam line and detector instrumentation would be the responsibility of university teams. 
TABLE JIIa

NTOF COST ESTIMATF, ${ }^{\text {A }}$

(Marerials, Fabrication)

$\begin{array}{lr}\text { Building Mods } & \$ 100,000 \\ \text { Beam Transport } & \$ 100,000 \\ \text { Swinger/Dump Crypt } & \$ 320,000 \\ \text { Beam Swinger } & \$ 420.000 \\ \text { Spin Precessor } & \$ 130,000 \\ 600-m \text { Flight Path and Hut } & \$ 3 j 1,000 \\ \text { Electronics } & \$ 200,000 \\ \text { Subtotal } & \$ 1,621,000 \\ & \\ \text { Contingency (25\%) } & \$ 405,000 \\ \text { ToTAL } & \$ 2,026,000\end{array}$

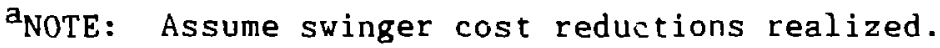

$b_{\text {Not }}$ required in minimal system.

TABLE IIIb

MRS COST ESTIMATES

(Materials, Fabrication)

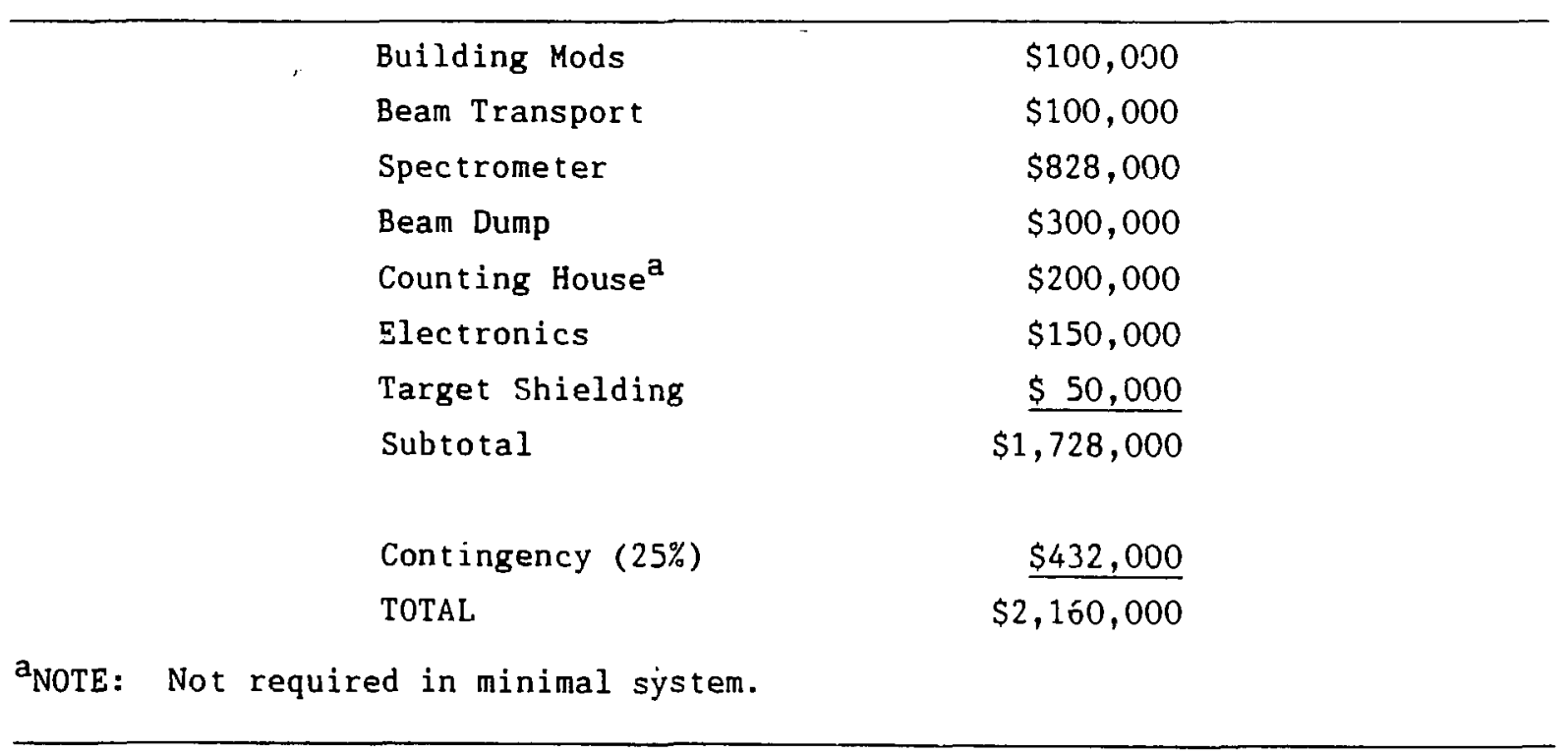


The time scale for a minimal MRS facility for $\left(\vec{p}, \vec{p}^{\prime}\right)$ measurements is estimated to be 1988. It would include a full focal plane detector array including focal plane polarimeter and a temporary counting house with electronics and computer. The cost of this system is given in Table IIIb. Costs are based on use of HRS style detectors and electronics and use of whatever computer is available at the time for the Area B area. Again, some parts of beamline and detectors would be the responsibility of university teams. Implementation of $(n, p)$ capabilities in BR would introduce minimal additional cost. It is estimated that an additional $\$ 250,000$ would be needed to implement an Area B $(n, p)$ targeting scheme. Table IV gives the time and manpower estimates for just the spectrometer.

TABLE IV

SPECTROMETER ESTIMATES

Cost

(Materials, Fabrication)

$\$ 828,000$

Manpower

10 Man-Years

Time

2.5 Years

MANPOWER (Man-Years)

Mech Eng

2.5

Design Draft

2.6

Elect Tech

1.2

Mech Tech

2.0

Mag Meas

0.5

P.S. Tech

0.4

Elect Eng

0.2

Elect Draft

0.2

Align

0 ?

P.S. Eng

TOTAL
0.2

10.0 
During the December workshop, many universities offered assistance in completing these projects. Most of the assistanre was in the form of design studies and fabrication of subsystems such as polarized target interface, generalized second arm, scattering chambers, vacuum systems, target shielding, detectors, beamline instrumentation, high count rate electronics systems, and software development. It was agreed that a LAMPF team would be responsible for overview o- all activities. As such, only major items ara included in the cost estimates. Even some of these items, however, might be turned uver to university teams.

\section{F. User Facility Aspects}

Suggestions were made during the December workshop regarding the user facility aspects of both the MRS and NTOF projects. It was felt that dedicated electronics and a countine house supporting a basic system would be necessary for both. The instrumentation would also be flexible enough to allow users to reconfigure it to adapt to new experimental requirements. Since the upgrade addresses both nucleon-nucleon and nucleon-nucleus physics programs, it was suggested that a representative NPL subcommittee (perhaps consolidated with HRS) would be responsible for generating a separate queue of experiments for each of the facilities. This would alleviate scheduling problems associated with ambiguities of priorities from different subcomittees. Once this apparatus is in place, a call for proposals should be made wher all proposals could be reviewed on the same footing. At the February 1985 meeting of the joint HRS/NPL subcommittee this question was addressed with the recommendation that scientific proposals for the MRS and NTOF facilities be reviewed by the NPL subcommittee and that it include more members with expertise in nuclear structure physics, particularly in the few-body problem.

It was felt important to proceed as quickly as posiible on both of these projects in order to take full advantage of the high intensity polarized source planned for 1988 which plays such a vital role in the programs being proposed.

G. Status of Proposal

i commitment has been made by the LAMPF management to implement the NPL upgrade within the next three years. A management structure has teen established and a scheine for funding the project has been devised. The development of the LAMPF facilities will be curcied out within the existing MP Division organization with general oversight by the Experimental Areas Development Committee (EADC). L. Agnew, Associate MP Division Leader for Experimental Areas, has been given the task for the overall management of the NPL upgrade program. $R$. Werbeck will be the technical coordinator with the 
following project managers: J. Donahue (MP-7)-Beamline Modifications (BLM) and HIRAB; J. B. MCClelland (MP-10)-NTOF; R. Boudrie (MP-10)-MRS. Users advisory panels will be formed to provide input from the scientific community and serve as a board of review for plans and options. The following individuals are recommended for the NTOF Advisory Panel: S. Austin, A. Bacher, C. Goodman, J. Moss, and $C$. Zaffiratos. The following individuals are recommended for the MRS Advisory Panel: G. Crawley, C. Glashausser, G. Glass, D. Lind, and J. Rappaport.

Preliminary cost estimates based on this proposal yield the following apprcximate cost estimates:

$$
\begin{array}{r}
\text { BLM - \$0.5M } \\
\text { NTOF - \$1.5M } \\
\text { MRS - \$2.0M } \\
\text { HIRAB - \$1.2M }
\end{array}
$$

These estimates cover outside design, hardware, and construction, but do not include the cost of MP Division manpower for design and assembly. Funds for NrOF, MRS, and BLM will come from several sources. Capital equipment money will be needed for the spectrometer and other major apparatus; Accelerator Improvement (AIP) funds will be used for changes in the permanent beamlines; and General Physical Plant (GPP) funds will be necessary for a counting house, building addition, and other construction.

\section{NEW PHYSICS PROGRIMS}

\section{A. Overview}

The facilities under consideration for the NPL upgrade will häve a signiflcant impact on both the nucleon-nucleon and nucleon-nucleus research programs at LAMPF. Studies of the nucleon charge-exchange reactions $(p, n)$ and $(n, p)$ with comparable resolution will provide a unique new probe of nuclear structure and of nuclear response functions. In addition, the MRS facility will allow new types of experiments to be initiated both in the area of nucleonnucleon interactions leading to multiparticle final states and in the area of proton interactions with light nuclei where the narrow acceptance of the HRS facility is a severe disadvantage.

The proposed facilities at LAMPF will operate in an energy range (200-800 MeV) that is uniquely suited to at tack fundamental open questions in nuclear structure physics. Within this energy range the nucleon probe can be tuned to emphasize particular features of the nuclear response. The specificity of the charge-exchange reactions, along with the complementary nature of the 
$(p, n)$ and $(n, p)$ reactions, will enable new types of nuclear excitations to be explored. In comination with existing and upgraded polarized-beam intensities at LAMPF, the experimental facilities will far surpass those available at all of the other accelerator laboratories in the world.

B. General Features of Nucleon Charge Exchange

The principal advantage of the $(p, n)$ and $(n, p)$ charge-exchange reactions involves the production of a pure isovector spectrum of final nuclear states. The dominant background of isoscalar nuclear states currently excited in ( $p, p^{\prime}$ ) studies at the HRS facility is eliminated. In addition, isospin coupling coefficients conspire to emphasize $\left(\mathrm{l}_{0}-1\right)$ final states in the $(\mathrm{p}, \mathrm{n})$ reaction and $\left(T_{0}+1\right)$ rinal states in the $(n, p)$ reaction. Taken tugether, the ability to measure $\left(p, p^{\prime}\right),(p, n)$ and $(n, p)$ reactions in the same laboratory will allow the spectrum of final nuclear states to be uniquely characterized.

There are several features of the underlying nucleon-nuclenn interaction that make the energy range available at LAMPF especially significant. Between about 100 and $500 \mathrm{MeV}$, the effective $\mathrm{N}-\mathrm{N}$ interaction which drives the nucleonnucleus reaction processes exhibics a strong dependence on spin. Above $500 \mathrm{MeV}$, spin-independent terms in the interaction again become stronger. This tunable nature of the selectivity of the nucleon probe nas an important impact on our ability to investigate specific features of the nuclear response function.

The excitation of known nuclear final states at several values of the momentum transfer $\mathrm{q}$ allows one to investigate modifications of the $\mathrm{N}-\mathrm{N}$ interaction in the nuclear medium. These medium modifications are expected to - change significantly over the range of energies available at IAMPF, and an understanding of how they change will have an imporiant impac: on our understanding of nuclear structure. At higher energies above about $400 \mathrm{HeV}$, the medium corrections are less important, the calibration of the nucleon probe is better understood, and the nucleon-nucleus reaction processes can be used directly to probe features of the nuclear response function.

In the calibration of the nucleon probe, nuclear structure acts as a filter to select or exclude components of the interaction and to allow one to map their q-dependences. For example, the structure overlap matrix element for a particular operator may be zero to a particular final state. This method of selection and exclusion has been exploited in nuclear beta decay to separate the Fermi (F) and Gamow-Teller (GT) coupling constants. Simply put, a $0^{+} \rightarrow 0_{+}$ transition goes by the $F$ interaction alone and a $0^{+} \rightarrow 1^{+}$transition goes by the GT interaction alone. The same idea has been applied to the study of the nucleon-nucleus interaction. Here the spin independent isovector component has 
been measured by studying isobaric analog transitions. Likewise, the spinisospin-flip component has been measured by studying excitations known to involve large GT transition matrix elements. Applying this same concept to a variety of states provides a means of mapping the q-dependence of certain interaction terms. : Without the multipolarity selection provided by the nuclear structure matrix element, observations at larger q would simp?y pick up higher transfer multipolarities and the q-dependence of a given force component would be masked.

Absorption of the nucleon probe is also an important consideration. Between 200 and $500 \mathrm{MeV}$ the absorption passes through a minimum and the nucleon probe is thus more sensitive to the nuclear interior. As might be expected, corrections due to distortion of the incoming and outgoing nucleon become better understood in this energy range. The rapid increase in absorption above $500 \mathrm{MeV}$ is also advantageous. It leads, for example, to a preference for exciting isovector monopole resonances in the charge-exchange reactions since contributions to radiai matrix elements are strongly affected. The stronger absorption at higher energies may have other advantages. One pion exchange processes become more significant in the underlying coupling to the nucleus leading, for example, to an enhanced sensitivity to the surface longitudinal spin response of the nucleus.

The higher energies available at LAMPF have a special significance for the extension of $(p, n)$ reaction studies initiated at lower energies at IUCF. If the missing GT strength identified in this work resides in $\Delta \mathrm{N}^{-1}$ components of nuclear wave functions, then the higher regions of the nuclear continuum accessible at LAMPF energies may provide a direct test of $N \Delta$ coupling assumed in these models.

\section{Specific Features if $(p, n)$ Charge Exchange}

Studies of the nucleon charge exchange $(p, n)$ reaction at energies below $200 \mathrm{MeV}$ have already provided beautiful and exciting new physics as evidenced by the extensive work coming from the Indiana University Cyclotron Facility (IUCF). This includes observation of low-lying giant resonances, particularly the GamowTeller giant resonance, mapping of the effective interaction at low momentum transfer, and spin-flip strength functions from depolarization measurements. However, many interesting aspects of the $(p, n)$ reaction are not accessible to 200-MeV protons.

There are several advantages of higher energy incident protons. The ability. to undergo much larger energy losses in the reaction is crucial in measurements of $(p, n)$ response functions where one would like to map the region 
up to $400 \mathrm{MeV}$ in order to study the effects of deltas in nuclei and still have reaction neutrons of sufficient energy for the impulse approximation to be valid. These studies are kinematically inaccessible at IUCF energies. Analyses of $\mathrm{N}-\mathrm{N}$ phase shift solutions indicate that major components of the effective interaction are changing dramatically from 200 to $800 \mathrm{MeV}$, the range of energies available at LAMPF. In particular, recent work on the ratio of isovector spindependent to spin-independent strengths shows tremendous variations depending on the assumptions made. Simple $(p, n)$ cross-section measurements at $0^{\circ}$ give this ratio directly. There is good evidence that the higher energies obtainable at LAMPF may be ideal for observation of isovector monopole resonances in the charg: exchange reaction and $(\vec{p}, \vec{n})$ measurements may provide the only irrefutable evidence for spin-flip isovector resonances.

We expect the major emphasis in the $(p, n)$ reaction to be placed on spin-transfer measurements. These measurements would include studies of lowlying discrete states, isovector giant resonances, and the nuclear continuum. LAMPF Research Proposal 881 addresses two of these aspects in the calibraticn of the neutron polarimeter and the quasi-free spin-transfer measurements using the polarimeter. Many details of the experimental procedures involved are discussed in that proposal. Nuch of the theory for the physics interpretation of the spin-transfer measurements has been developed for the interpretation or $\left(\vec{p}, \vec{p}^{\prime}\right)$ reaction studies at the HRS over the same energy range and can be directly applied to the $(\vec{p}, \vec{n})$ reaction. The additional exclusiveness gained by using the $(p, n)$ reaction should allow us to make more incisive comparisons with theory. Combining $(p, n)$ with spin-flip measurements allows us to select the $\Delta T=1$, $\Delta S=1$ channel. The extensive $(p, n)$ program at IUCF has already provided many clues to the physics opportunities from using this reaction. In addition, cross-section measurements at WNR (primarily at $800 \mathrm{MeV}$ ) confirm our beliefs both that the reaction mechanism is simple and that the experimental technique is applicable up to $800 \mathrm{MeV}$.

In the impulse approximation, specific combinations of the spin-transfer observables are proportional to individual spin-dependent amplitudes of the nucleon-nucleon (N-N) scattering matrix. In this manner, a complete determination of spin-transfer observables allows a very selective mapping of the effective nucleon-nucleus interaction to be made. Exclusive measurements selecting the different spin and isospin components to compare to effective interaction calculations have been carried out at the HRS in a limited number of cases using the predominantly isoscalar ( $\left.p, p^{\prime}\right)$ reaction. Improvements in the detector shielding at the HRS should provide more accurate systematic studies to 
be carried out in the near future. Intercomparison of the $(p, n)$ and $\left(p, p^{\prime}\right)$ spin-transfer daia will provide an espectally incisive test of thenretical predictions.

Once ertablished, the theoretical formalism can be applied in a number of areas where the nuclear structure is less well-determined. The ability to separate complex transitions into $\Delta S=0$ and $\Delta S=1$ modes allows one to examine the distributions of both Fermi (F) and Gamow-Teller (GT) strength. For low-lying discrete transitions, spin-transfer measurements will provide a technique for identifying composite nuclear currents (e.g., convection spin) which are difficult to excite with other probes such as (e,e'). Combinations of observables such as $(P-A)$ and $\left(D_{S L^{\prime}}+D_{L S^{\prime}}\right)$ are sensitive to currents of the form $(i \vec{L} \times \vec{\sigma})$ which have previously been accessible only in measurements of B-decay.

In studies of the nuclear continuum, the structure selectivity cannot be exploited, and spin-transfer measurements may be the only way to regain some measure of exclusivity. Thus, one selects the spin-isospin component by using a beam that is polarized in both spin and isospin and by measuring both the spin and the isospin polarization of the outgoing nucleon. Fortunately, the isospin polarization comes free. Unfortunately, we have to work hard to create and measure spin polarization.

An important series of measurements compares spin transfer in the nucleon-nucleus charge exchange scattering with that in the nucleon-nucleon charge exchange channel. This comparison will tell us to what extent we can characterize the nuclear response (including the delta region) on the basis of independent particle motion and to what extent many-body effects modify the response. The detailed LAMPF Research Proposal 881 addresses this question in the quasi-free nucleon sector specifically as it applies to the European Muon Collaboration (EMC) effect. Because of the large energy losses involved (typically 30 to $130 \mathrm{MeV}$ ), interpretation of this type of experiment requires the higher energies obtainable at LAMPF.

Studies with the $(p, n)$ reaction in the energy range of 100-200 MeV at IUCF have shown that the total GT strength contained in the excitation region of ordinary nuclear particle-hole states is substantially less than the minimum required by the GT sum rule. Inasmuch as the sum rule is an exact operator relationship, the lack of saturation of the sum rule must indicate that either the energy region over which the GT strength is integrated is not the complete region over which the strength is distributed, or that the strength is dispersed so thinly that it is indistinguishable from experimental background. 
Two mechanisms for redistribution and dispersal of the GT strength that have ceceived considerable attention in the recent literature are: (1) mixing of the simple GT states into states of nucleon-hole, delta-particle character and (2) mixing of the simple GT states into the highly numerous states of multiparticle multi-hole character. The two pictures differ in the expected distribution of strength, particularly out to the region of delta excitation $(-300 \mathrm{MeV})$.

Both the $(p, n)$ and $(n, p)$ reactions can be calibrated directly to beta decay to obtain $B(G T)$ values without relying on reaction calculations. The reactions are then used to extend measurements into regions energetically excluded to beta decay. Spin-flip cross-section measurements allow one to distinguish between GT and $F$ strength and hence to deduce the $B(G T)$ strength functions. The need for LAMPF energies is obvious when one is looking at up to $400-\mathrm{MeV}$ energy losses in mapping the delta region. Studies of the beta strength functions and associated spin-flip probabilities should be accessible as soon as the $(p, n)$ phase is on-line.

The general question of isovector resonances can be well addressed by both the $(p, n)$ and the $(n, p)$ raactions at LAMPF energies. By utilizing the strong energy dependence of the ratio of spin-isospin to isospin pieces of the force, one may enhance and discriminate between spin-flip and nonspin-flip giant resonances. In the $(\vec{p}, \vec{n})$ reaction, one can also take advantage of spin-flip cross-section measurements to differentiate between the two types of giant resonances. Since predictions of the positions of these excitations depend strongly on the form of the residual particle-hole interaction in nuclei, their identification will provide a siringent test of existing models of nuclear structure.

LAMPF is considered the birth place of intermediate-energy nucleun-nucleus relativistic models. It was in fact polarization transfer $(\vec{p}, \vec{p})$ data from HRS which provided the basis for such an interpretation. We feel that the polarized aspects of the proposed $(\vec{p}, \vec{n})$ facility provide truly unique features which will keep LAMPF at the forefront of these investigations. Complete sets of purely isovector polarization-transfer observables similar in quality to the $(\vec{p}, \vec{p})$ work being done at HRS at the same energies will be possible via the $(\vec{p}, \vec{n})$ reaction. This comparison of $(\vec{p}, \vec{n})$ and $\left(\vec{p}, \vec{p}^{\prime}\right)$ data is essential in sorting out the isoscalar/isovector nature of the proton-nucleus interaction.

The relativistic impuise approximation (RIA) approach has been particularly effective in removing discrepancies between experiment and nonrelativistic distorted wave impulse approximation (DWIA) predictions for forward-angle spin 
observables. Clark et al. have emphasized that elastic scattering studies have thus far tested only the isoscalar parts of the linrentz scalar and vector terms in the $\mathrm{N}$-nucleus relativistic optical potential. These authors suggest comparisons between RIA and DWIA predictions and $500-\mathrm{MeV}(p, n)$ data for the excitation of the isobaric analogue states from spin-0 targets with filled $(l, j)$ subshells (e.g., ${ }^{90} \mathrm{Zr}$ ). Such comparisons should enable us to directly determine the isovector components of these potentials. The most dramatic conseqliences of a relativistic description of the $(p, n)$ reaction appear in the structure of the spin-transfer observables. Studies of polarization transfer in intermediate energy $(p, n)$ will thus provide new knowledge regarding the importance of relativistic effects in the general description of $\mathrm{N}$-nucleus scattering.

\section{Specific Features of $(n, p)$ Charge Exchange}

In sharp contrast to the $(p, n)$ reaction, intermediate energy studies of the $(n, p)$ charge exchange reaction at the proposed level of resolution (1-2 MeV) are nonexistent. The selectivity of $\left(\mathrm{T}_{0}+1\right)$ final state nuclear excitations has been observed via studies of the $\left(\pi^{-}, \pi^{0}\right)$ and $\left(t,{ }^{3}\right.$ He) reactions but this work is limited aither by poorer resolution or by the complexity of dealing with composite projectiles in the charge-exchange process.

The first generation of $(n, p)$ experiments, for reasons of technical feasibility, will not measure spin rransier, but will use a nuclear-physicsgrade unpolarized neutron beam and the MRS to detect the charge-exchange protin. In the $(n, p)$ reaction, emphasis is placed on cross-section measurements of lowlying discrete transitions and isovector giant resonances. LAMPF Research Proposal $823 \mathrm{U}$ contains many of the experimental details. In comparison with $(p, n)$ measuremencs on the same target, the linique separation and identification of $\left(\mathrm{T}^{0}+1\right)$ and $\left(\mathrm{T}^{0}-1\right)$ states will provide important new constraints on current nuclear shell models.

of special importance is the fact that $(n, p)$ charge-exchange measurements will allow a direct test to be made of sum rule predictions for the GT giant resonance. The simplest approach gives $\mathrm{S}_{\mathrm{pn}}^{\mathrm{GT}}-\mathrm{S}_{\mathrm{np}}^{\mathrm{GT}}=3(\mathrm{~N}-\mathrm{Z})$.

In the fnormal nucleon model, most of the beta ${ }^{+}$strength (obtained from $(n, p)$ studies) is Pauli blocked because in nuclei with neutron excesses the states into which protons would be taken are already occupied. In the delta model, two mechanisms create beta ${ }^{\dagger}$ strength. GT transitions from protons to deltas are not Pauli blocked, so a good deal of beta ${ }^{+}$GT strength would appear in the delta region of the spactrum. Also, the mixing tends to create neutron vacancies in the nucleon states. In heavy nuclei the $\Delta \mathrm{N}^{-1}$ model predicts an enhancemen: in $\mathrm{S}_{\mathrm{np}}$. 
Precise measurements of the beta ${ }^{+}$strength functions using the (n,p) reactions might well be the most directly interpretahle data that could be obtained in refation to the observed missing Gr strength. It is also possible that simple cross-section measurements will not be sufficiently exclusive. Ultimately, spin-flip measurements will be required to distinguish isovector monopole transitions from GT transitions. These are considered to be secondgeneration experiments for $(n, p)$ because of the high polarized-neutron currents required when focal-plane polarimetry is requi $-d$.

\section{ACKNOWLEDGMENTS}

This report represents the views of a variety of LAMPF users and staff. Their enthusiasm is responsible for the rapid progress of this proposal. We wish to thank ail of those contributing to the workshop and in particular G. Love and Amir Klein (University of Georgia), D. Lind and J. Shepard (University of Colorado), G. Crawley (MSU), G. Igo (UCLA), H. Spinka (Argonne), G. Glass (Texas A\&M University), G. Hoffmann (University of Texas, Austin), C. Glasshauser (Rutgers University), and J. Moss (Los Alamos). 
APPENISIX A

BEAM SWINGER SYSTEM FOR STUDYING $(p, n)$ RFACTIONS AT LAMPF

In order to carry out extensive, high resolution $(p, n)$ studies at LAMPF, it is necessary to vary the scattering angle at the production target and detect the scattered neutrons at a large distance from the targ*t. The latter requirement indicates the need for long neutron flight paths (up to $600 \mathrm{~m}$ ) and large detector systems $(1 \mathrm{~m} \times 1 \mathrm{~m})$. The former lequirement can be met in two ways; either construct several flight paths at various angles relative to the incident proton beam or construct one neutron flight path and devise a system to change the angle at which the incident protons strike the production target. It is more economical and practical to perform the second option, i.e., constluct a system to "swing" the proton beam on the target, thus varying the angle between the incident protons and the fixed neutron flight path.

The swinger system which has been designed for LAMPF will achieve momentum transfers of at least $3 \mathrm{fm}^{-1}$ in the energy range from $200 \mathrm{MeV}$ to $800 \mathrm{MeV}$. Figure A.1 indicates the relationship between the maximum attainable momentum transfer at a given beam energy and incident proton beam energy. Figure A-2 is the planned layout for the swinger system. It was desigred to utilize existing, surplus magnets and radiation shielding wherever possible. The system consists

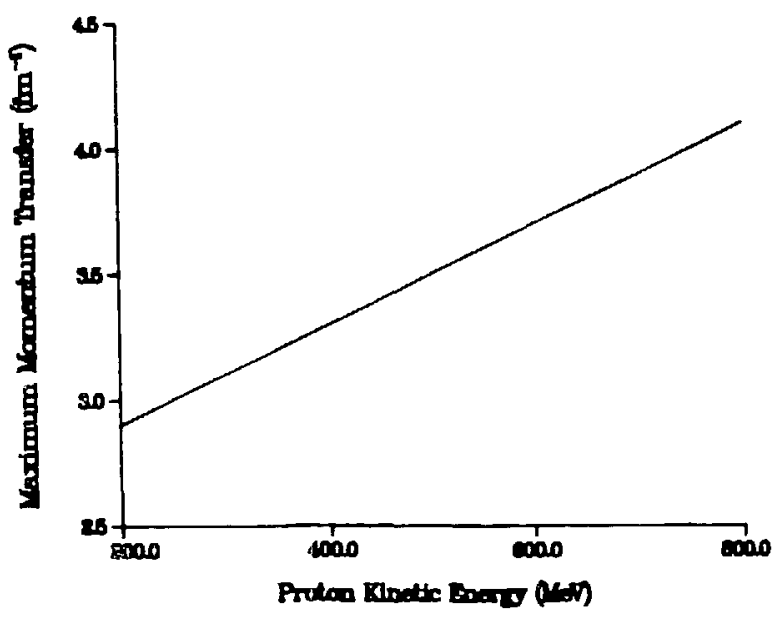

Fig. A-1

Momentum transfer vs. energy for swinger. 


\section{$(p, n)$ SWINGER}

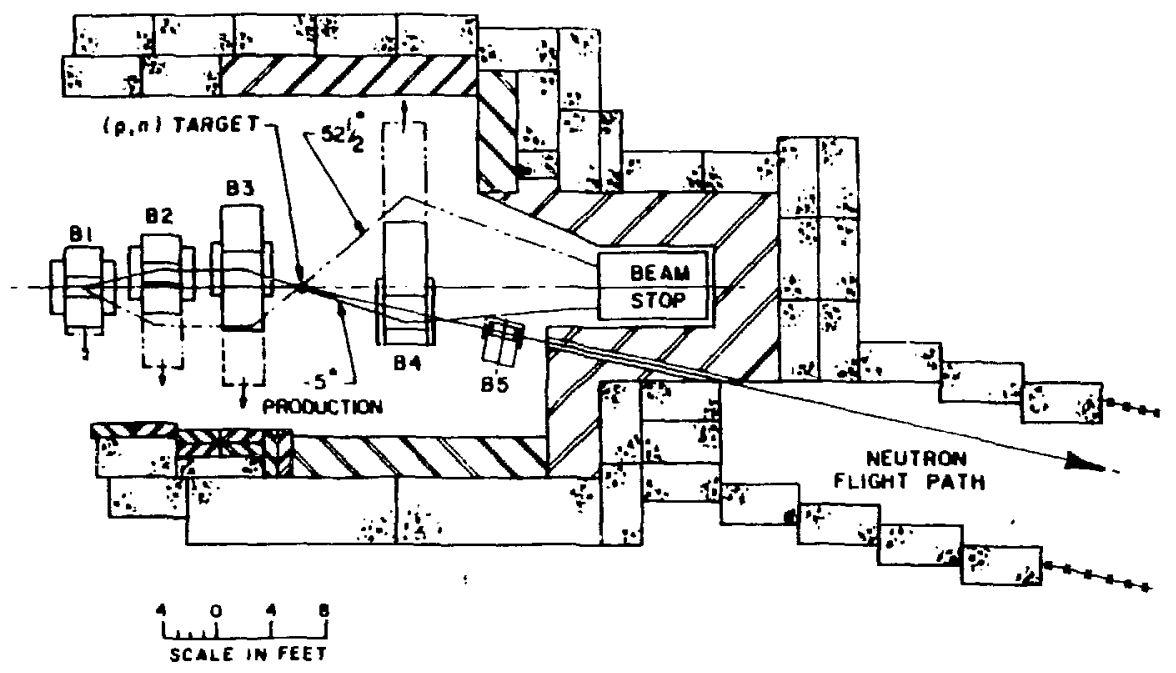

Fig. A-2

The $(p, n)$ swinger and crypt.

of a shielded "crypt," three dipole bending magnets (B1, B2, B3) which vary the angle at which the incident beam strikes the $(p, n)$ target, a fourth dipole magnet (B4) which directs the residual proton beam to a large graphite beam stop, and a fifth bending magnet (B5) which serves as a charged particle sweep magnet, a neutron spin precessor, and a part of the personnel safety system. Also included are the vacuum systems (including the scattering chamber which contains the target), beam diagnostics, neutron collimators, neutron spin precession magnets (outside of the crypt), support structures for the magnets, and magnet power supplies.

It should be noted that to minimize the excavation required to construct the neutron flight path east of the access road leading to the NPL, inclining the flight path upward from the $(p, n)$ target at about $1 / 2^{\circ}$ is highly desirable. To achieve true $0^{\circ}$ neutron production, the incident proton beam must be inclined 
at the same angle. Thus the entire beamline downstream of the $7^{\circ}$ bending magnet which delivers beam to the swinger will be inclined upward at the approprlate angle; the beam will be directed upward by a small dipole bending magnet. This means that the whole swinger system will be tilted upward along this inclined beamline.

If the proton beam delivered by the switcher magnet system is undeflected by the swinger magnets (i.e., protons follow the axis of the swinger), it will strike the production target at an angle of $12-1 / 2^{\circ}$ with respect to the neutron flight path; i.e., with the first three bending magnets of the swinger set to zero magnetic field, $12-1 / 2^{\circ}$ neutron production (scattering) is achieved. By deflecting the proton beam to the left with B1 and by using B2 and B3 to bend the bean to the right so that the protons which strike the $(p, n)$ target are collinear with the neutron flight path, $0^{\circ}$ production is possible. Thus a net proton bend of $12-1 / 2^{\circ}$ to the right is required for $0^{\circ}$ scattering; this also requires that $B 4$ be set to bend the residual proton beam to the left into the beam stop. When the magnetic field directions of $B 1, B 2, B 3$, and $B 4$ are set as they are for $0^{\circ}$ production (so that $B 1$ deflects the beam to the left), scattering angles from $-5^{\hat{b}}$ to $+12-1 / 2^{\circ}$ are attainable; when their magnetic field directions are reversed ( $B 1$ bends protons to the right), scattering angles from $+12-1 / 2^{\circ}$ to $+52-1 / 2^{\circ}$ can be reached. Because of the physical reality of the current carrying coils of $B 4$ which cannot be allowed to intercept the transmitted proton beam and because of the limited width of the beam stop $(1.5 \mathrm{~m})$, there are two small angular "dead spots" (from $+7-1 / 2^{\circ}$ to $+10^{\circ}$ and from $+15^{\circ}$ to $+12-1 / 2^{\circ}$ ) in the range of neutron production angles which cannot be realized by the swinger system. Aside from these two forbidden angular regions, neutron scattering angles (in the laboratory reference frame) in the angular range from $-5^{\circ}$ to $+52-1 / 2^{\circ}$ are possible.

All the magnets to be used in the swinger system are existing, surplus magnets. The pole tip widths of $B 1$ and $B 2$ are $0.45 \mathrm{~m}$, and those of $B 3$ and $B 4$ are $0.75 \mathrm{~m}$. However, in $\mathrm{B} 2$ and $\mathrm{B} 3$, the particle trajectories move from $0.40 \mathrm{~m}$ to the left of the swinger axis (for $-5^{\circ}$ production) to $0.95 \mathrm{~m}$ to the right (for $+52-1 / 2^{\circ}$ production). In B4, the corresponding spatial deflections ate $0.75 \mathrm{~m}$ to the right (for $-5^{\circ}$ ) and $1.90 \mathrm{~m}$ to the left (for $+52-1 / 2^{\circ}$ ). Such variations in the left-right extents of particle trajectories require that the first four swinger magnets be on support structures which allow rather large (up to $2.25 \mathrm{~m}$ ) horizontal motion perpendicular to the swinger axis. The requirement for such large motions and the need to incline the entire swinger system make the design of the magnet support structures quite complex and costly. Because of the large 
aperture magnets and extensive magnet motions planned for the swinger, horizontal aperture is not the limiting factor in determining the maximum achievable momentum transfers. The most significant factor is the maximum magnetic field possible in B4. Momentum transfer calculations have been made assuming a maximum fieid of $18 \mathrm{kG}$ in $\mathrm{B} 4$.

At all scattering angles, the neutrons pass through a well defined magnetic region of $\mathrm{B5}$; in the production angle range fiom $-5^{\circ}$ to $+7-1 / 2^{\circ}$, neutrons also pass through well defined regions of $B 4$. With a clear definition of the magnetic fields through which the production neutrons traverse, consideration of neutron spin precession through B4 and B5 is not a problem. Further neutron spin precession to occur outside of the swinger crypt (downstream of the neutron collimator) must be fully addressed. At present, a superconducting solenoid and one or two more bending magnets are being considered. All such magnets will hopefully be existing, surplus magnets. The beam protons which are transmitted through the produciion target are bent by $B 4$ into the wide graphite beam stop. By allowing these protons to merely strike the beam stop at some location, and b; :-.jt requiring a "focus," smaller bend angles are required to "restore" the proton beam. Since the maximum magnetic field in B4 is our limiting factor in the swinger system, smaller restoration angles result in larger possible production angles.

At present, issues regarding swinger magnets, magnet support structures, and the shield crypt are basically resolved. Details of the vacuum systems (including scattering chamber), beam diagnostics, neutron collimators, spin precessors, and magnet power supplies must be addressed, although some preliminary work has been carried out on these items. Table A-I contains a breakdown of estimated costs for the swinger system. 
TABLE A-I

SWINGER/PRECESSOR COST ESTIMATES*

(Materials, Fabrication)

MAGNETS :

$\mathrm{B1}, \mathrm{B} 2, \mathrm{~B} 3, \mathrm{~B} 4, \mathrm{~B} 5$

Neutron Spin Precessors

0

MAGNET POWER SUPPLIES (installed):

$\mathrm{B} 1, \mathrm{~B} 2, \mathrm{~B} 3, \mathrm{~B} 5$

B4

4 ea 250

200

Spin Precessors

2 ea e 50

60

1 ea 920

100

20

MAGNET SUPPORT STRUCTURES :

B1, B2, B3, B4, B5

100

Spin Precessors

20

VACUUM SYSTEM:

80

SHIELDING :

Concrete Slab

Crypt

Beam Stop

40
120

100

NEUTRON COLLIMATORS:

40

ENCLOSURE (weatherproof): $20 ?$

DIAGNOSTICS (Beam Profile Monitors (BPM), polarimeters, etc.):

CRYOGENICS :

Superconducting Magnets

50

CONTROLS (RP, PSS, interlocks):

70

UTILITIES (power, water, heat):

100

TOTAL

$\$ 1,150$

ॠNOTE: Drastic reductions in power supply costs and full reimbursement
for the beam stop may be realizable. 